\title{
La presencia de producción científica española en la geografía de los países de América Latina y El Caribe en el cambio de milenio'
}

\author{
Francisco Cebrián Abellán² y Carmen Delgado Viñas ${ }^{3}$
}

\begin{abstract}
RESUMEN
Desde principios de la década de los noventa del siglo XX se ha producido un incremento y diversificación en España de la producción científica en Geografía acerca de los países iberoamericanos. En el texto se han identificado, por una parte, los artículos de investigación editados en las revistas de Geografía y por otro lado las tesis doctorales. Los trabajos se han clasificado bajo criterios territoriales y temáticos. Desde el punto de vista espacial se han agrupado en grandes unidades regionales, por un lado, y en países, por otro. En cuanto a orientación temática, se han clasificado por grandes temas y en subtemas. El resultado del análisis ha permitido identificar cómo ha evolucionado el número de trabajos, las disciplinas y territorios que han tenido un protagonismo mayor, así como los efectos de las diferentes coyunturas y estrategias institucionales en relación con los países hispanoamericanos.
\end{abstract}

Palabras clave: Geografía de Iberoamérica en España, tesis doctorales de geografía Iberoamérica en España, revistas españolas de geografía

\begin{abstract}
From the beginning of the last decade of the twentieth century, there has been an increase and diversity in the production of scientific geographic inquiry in Spain concerning Latin America, both in terms of the number of research articles published in Geogaphic journals and in doctoral theses. The works have been classified according to territorial and thematic criteria. Territorially, works have been grouped either by large regional units or by nation states. As for thematic orientation, large themes have been grouped and sub-themes identified. The result of this classification allowed to identify the number of works that have evolved and the diverse disciplines and territories that have had the most impact on the institutional strategies and conjunctures in the work done on Latin American.
\end{abstract}

Key words: Geography of Latin America in Spain, Doctoral Thesis in Geography of Latin America, Spanish Geographic journals

Artículo recibido el 3 de abril de 2017, aceptado el 20 de diciembre de 2017 y corregido el 30 de enero de 2018 . Universidad de Castilla-La Mancha (España). E-mail: Francisco.Cebrian@uclm.es

Departamento de Geografía, Urbanismo y Ordenación del Territorio, Universidad de Cantabria (España)

E-mail: carmen.delgado@unican.es 
La evolución de Geografía española ha sido especialmente significativa en las últimas cuatro décadas. En este periodo, aunque con altibajos, se han incrementado los efectivos demográficos universitarios (estudiantes y profesores), en buena medida por el aumento y dispersión del número de universidades y titulaciones (Gómez, 2013: 24). En paralelo se ha venido produciendo un cambio epistemológico y conceptual de la disciplina, acelerándose el paso de los estudios de carácter local, muy influidos por la tradición geográfica francesa, a otros territorios, incorporando nuevos enfoques teóricos y metodológicos. Ha sido parte del proceso de maduración de la disciplina, en un periodo definido por el eclecticismo, la necesidad de renovación y la influencia de otras escuelas, en especial la anglosajona y la latinoamericana. Es importante entender en este contexto el esfuerzo internacionalizador, los ámbitos de interés territorial y los principales actores en el proceso. En esa dirección se presenta este trabajo, con la mirada puesta en Iberoamérica, hacia donde un nutrido grupo de investigadores han dirigido su atención desde enfoques e intereses diversos.

Es un reto complejo intentar sintetizar en un texto breve las contribuciones recientes de la Geografía española a los estudios en Iberoamérica y el Caribe. La razón es evidente: lo producido es mucho, los repositorios y los formatos bibliográficos en los que se han editado diversos, y la naturaleza de las investigaciones muy variada en lo temático y lo territorial. En el proceso han participado profesores universitarios, investigadores y profesionales desde planteamientos teóricos y epistemológicos diferentes.

La producción científica ha sido especialmente intensa en los últimos veinticinco años. Hasta ese momento han existido relaciones personales e institucionales entre geógrafos y geógrafas españoles y latinoamericanos, pero es desde comienzos de los noventa cuando aparecen estructuras organizadas y sobre todo se incrementan en número y en trascendencia. En ellas ha tenido un especial protagonismo la creación de la Agencia Española de Cooperación Internacional, la Fundación Carolina, los programas Alfa o los Erasmus Mundus. También han proliferado los apoyos a la investigación desde las diferentes Comunidades Autónomas españolas. A todo se ha sumado la tarea realizada por las universidades españolas, a través de sus departamentos de Geografía, que han abierto vías de colaboración con sus homólogos latinoamericanos. Como resultado de este interés creciente se han financiado y apoyado económicamente numerosos proyectos y trabajos de investigación interuniversitarios e internacionales, actividades de cooperación al desarrollado, congresos y reuniones científicas. A todo ello hay que añadir la creación de redes y grupos de investigación a ambos lados del Atlántico, que han desarrollado iniciativas comunes. Una prueba fehaciente del fruto de esta intensificación ha sido también la puesta en marcha de programas conjuntos de formación universitaria de maestría y doctorado o la aparición de algunos centros de investigación especializados. Se suma a todo ello la organización de eventos y reuniones científicas, liderados por la Asociación de Geógrafos Españoles y sus Grupos de Trabajo, en particular del Grupo de América Latina (Carpio, 2013), o por el Grupo GeoCrítica.

Entre todos han creado el soporte académico para la elaboración de trabajos de investigación, muchos de los cuales se han materializado en forma de tesis doctorales (defendidas por doctorandos españoles e hispanoamericanos en la práctica totalidad de los programas de doctorado habilitados por las universidades españolas), de publicaciones de artículos en revistas especializadas, de participación en congresos y seminarios y edición de trabajos monográficos y capítulos 
de libro sobre diferentes temáticas relacionadas con la Geografía de Iberoamérica (en los que han participado activamente investigadores de ambos lados del Atlántico).

Las últimas décadas pueden considerarse como las que más positivamente han influido en la conformación de un modelo universitario español. Algunos trabajos han prestado atención al periodo de génesis y conformación de la Geografía académica entre las décadas de los cincuenta a setenta (Capel, 1976). Entre 1970 y 2010 se ha producido el aumento más importante en el número de universidades en nuestro país, multiplicándose por cuatro (Valenzuela, 2013). La disciplina geográfica ha salido beneficiada en este proceso. En el año 2017 existen 26 Grados en Geografía (con los nombres de "Geografía y Ordenación del Territorio"; "Geografía"; "Geografía y Gestión del Territorio"; "Geografía, Ordenación del Territorio y Gestión del Medio Ambiente"; "Geografía y Medio Ambiente"), a los que se añade otro en el que está muy presente nuestra disciplina: se trata de los Grados en "Geografía e Historia" (http://www.age-geografia.es/site/ensenanza-universitaria-grados/). A ellos se suman otras titulaciones en las que la Geografía tiene mucha presencia como disciplina, aunque no es su eje medular (es el caso de los Grados en Humanidades, Medio Ambiente, Educación...). Junto a los estudios de Grado se han implantado másteres y doctorados que han sido el canal de especialización profesional y sobre todo de investigación básica y aplicada. En este contexto se produce la mayor parte de la producción científica geográfica. Simultáneamente han surgido algunas asociaciones vinculadas al ámbito disciplinar de la Geografía, entre las que ha tenido un protagonismo especial la Asociación de Geógrafos Españoles desde 1975.

El profesorado universitario está adscrito a cuarenta y cinco Departamentos de otras tantas universidades, con docentes e investigadores de las áreas de Geografía Física, Geografía Humana y Análisis Geográfico Regional. Hay que añadir la producción surgida en el sector privado, con un colectivo de profesionales que, desde 1999, se agrupan mayoritariamente dentro del Colegio de Geógrafos. También destaca la labor realizada por docentes e investigadores de disciplinas con intereses temáticos afines a la Geografía.

A este proceso han prestado atención algunos trabajos que se han centrado en diferentes territorios, en distintas escalas de análisis y bajo los diversos enfoques y planteamientos teóricos. Casi todos han empleado los repertorios bibliográficos como forma de medir la cantidad y tipología de las contribuciones de la Geografía española. También han sido diversos los medios de difusión, tanto en España como en diferentes países iberoamericanos. (Cabero, 1993; Espejo, 1997; Gutiérrez, 1999; Mendoza, 2001; Panadero, 2004; Olcina y Román, 2004; García et al., 2009; Gurría et al., 2013; Cebrián, 2013; Valenzuela, 2013).

En este caso se ha optado por establecer una metodología que sigue o continúa con la línea de las referencias bibliográficas indicadas anteriormente, pero con matizaciones y, sobre todo, con una mayor intensidad de análisis. Por ello, para apreciar los resultados de estas relaciones crecientes, centraremos la atención en la cantidad y tipología de trabajos reproducidos en las revistas españolas, así como en la defensa de tesis doctorales en los diferentes programas de doctorado liderados por departamentos y universidades en España. El trabajo se ha realizado desde un enfoque analítico-descriptivo, sin entrar en considerar los enfoques teórico y metodológicos utilizados en cada uno de ellos. Se ha centrado la atención en el periodo comprendido entre 1990 y 2016. 


\section{El contexto en el que se inscribe la producción científica de la Geografía Española}

\section{Propuesta metodológica}

Para la confección de este trabajo se han seleccionado como elementos objetivos de análisis dos variables. Por una parte, las tesis doctorales defendidas en los diferentes Departamentos de Geografía de las universidades españolas (la información se ha extraído de la base de datos Teseo (https://www.educacion.gob.es/Teseo). Por otro lado se ha empleado la relación de revistas españolas de Geografía que aparecen incluidas en el listado de RESH (Revistas Españolas de Ciencias Sociales y Humanidades), que es un sistema de información que integra indicadores cualitativos y cuantitativos de calidad (utilizado por la CNEAI -Comisión Nacional Evaluadora de la Actividad Investigadora de España-, ANECA -Agencia Nacional de Evaluación de la Calidad Española- y Latindex en sus respectivos sistemas de evaluación para las revistas científicas españolas de Ciencias Sociales y Humanidades).

Se ha entendido en este caso que el papel de las revistas es especialmente representativo de la producción científica española. La modernización y consolidación de la Geografía en España ha venido marcada por la aparición de un nutrido grupo de publicaciones periódicas especializadas, que son referentes obligados en la investigación. Junto a los congresos, que periódicamente se dedican a las diferentes temáticas de la Geografía, generan una parte muy importante del repertorio bibliográfico que refleja el resultado de su aportación científica dirigida a Hispanoamérica y el Caribe.

Las revistas españolas precursoras se remontan a finales del siglo XIX (Boletín de la Real Sociedad Geográfica) y a la primera mitad del siglo XX (Estudios Geográficos -1940-, Geographica -1954-). Un segundo grupo lo constituye las que surgieron en las décadas de los años sesenta y setenta del siglo XX, cuando se consolidaron los estudios de esta disciplina y nacieron muchos de los actuales Departamentos de Geografía de las grandes universidades españolas, que impulsaron nuevas revistas como seña de identidad y como medio de difusión de su producción científica. El tercer momento coincide con la década de los años ochenta y noventa del pasado siglo, en el que aparecieron nuevas universidades y se produjo un crecimiento de las titulaciones y de las licenciaturas en Geografía (convertidas a comienzos del siglo XXI en Grados) (Olcina y Román, 2004: 150). Como resultado quedan más de cincuenta revistas en activo (dentro del catálogo de RESH se incluyen cincuenta y seis revistas en la categoría de Ciencias Sociales: Geografía) (http:// epuc.cchs.csic.es/resh/que_es) (Cuadro $N^{\circ} 1$ ).

Cuadro $N^{\circ} 1$

Revistas de Geografía Catálogo RESH

\begin{tabular}{|l|}
\hline Ager. Revista de Estudios sobre Despoblación y Desarrollo Rural \\
\hline Alisios. Revista de Geografía \\
\hline Anales de Geografía de la Universidad Complutense \\
\hline Ar@cne \\
\hline Baetica. Estudios de Arte, Geografía e Historia \\
\hline
\end{tabular}


Continuación Cuadro N¹

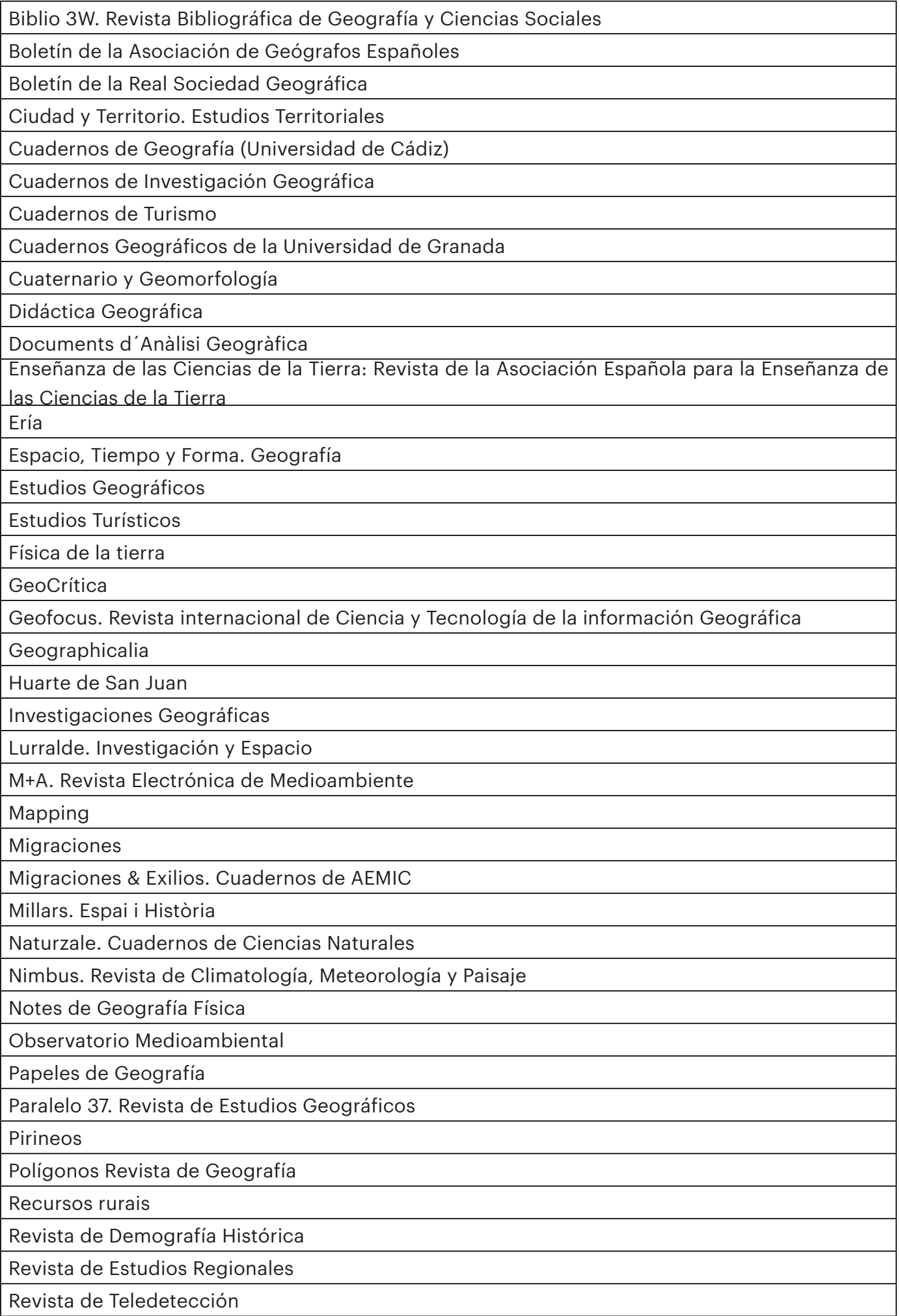


Continuación Cuadro $N^{\circ} 1$

\begin{tabular}{|l|}
\hline Revista Española de Estudios Agrosociales y Pesqueros \\
\hline Revistes Catalanes amb Accés Obert (RACO) \\
\hline Scripta Nova. Revista Electrónica de Geografía y Ciencias Sociales \\
\hline Serie Geográfica \\
\hline Terra: Revista de Desarrollo Local \\
\hline Territoris. Revista del Departament de Ciencies de la Terra \\
\hline Topografía y Cartografía. Revista del llustre Colegio Oficial de Ingenieros Técnicos en Topografía \\
\hline Treballs de la societat Catalana de Geografía \\
\hline Vasconia. Cuadenos de historia-geografía. \\
\hline Vegueta. Anuario de la Facultad de Geografía e Historia \\
\hline Xeográfica. Revista de Xeografía, Territorio e Medio Ambiente \\
\hline
\end{tabular}

Fuente: http://epuc.cchs.csic.es/resh/indicadores

Conviene señalar que no todas ellas tienen como campo disciplinar específico la Geografía, ya que en ocasiones su línea editorial comparte objetivos con otros ámbitos de las Ciencias Sociales, de las Ciencias de la Tierra y las Humanidades (e.g. Demografía, Cartografía, Turismo, Urbanismo, Estudios Territoriales, Teledetección, Sociología, Desarrollo Rural, Medio Ambiente) (Olcina y Cantos, 2004: 150). Tampoco poseen todas ellas los mismos criterios de calidad y de difusión (http://epuc.cchs.csic.es/resh/indicadores); prueba de lo cual es que solamente dos (Boletín de la Asociación de Geógrafos Españoles y Scripta Nova) cuentan en 2017 con la condición de JCR -Journal Citation Reports-, la práctica totalidad aparecía indexada en el año 2015 en diferentes bases de datos y sólo seis no estaban indexadas en ninguna base de datos bibliográfica (http:// epuc.cchs.csic.es/resh/que_es).

Los ámbitos espaciales de análisis han sido, sobre todo, los propios de las universidades en que se generaban (Valenzuela, 2000) en forma de estudios regionales, comarcales o locales, justificado en que la investigación aplicada se ha hecho, en su mayor parte, dentro de las demarcaciones político administrativas de las respectivas universidades. Asimismo, los trabajos han sido más numerosos para los espacios con afinidades socioculturales.

También se ha producido una diversificación y especialización temática, porque la disciplina ha ido abriendo su abanico epistemológico, y eso ha supuesto un incremento en la producción científica publicada en las revistas, que se han ido acomodando a las nuevas realidades. Han sido en definitiva años de consolidación de la disciplina en la Universidad española, que se ha materializado en la proliferación de estudios empíricos sobre temáticas paulatinamente más variadas. Pero cada vez es más frecuente encontrar trabajos dedicados a territorios más alejados, y que en ocasiones trascienden las fronteras nacionales, contribuyendo así a la consolidación e internacionalización de las revistas.

Queda, por tanto, un escenario enriquecedor y desigual en las revistas en cuanto a los parámetros de calidad habitualmente empleados y a los intereses territoriales de análisis. Por ello se ha realizado una revisión de todas ellas, asumiendo que se puede dar la circunstancia de que algunos de los artículos reproducidos en ellas están dirigidos a escenarios e intereses no coinciden- 
tes con el colectivo de investigadores en Geografía. De igual modo existe producción científica sobre Hispanoamérica, realizada por geógrafos y geógrafas, que se ha editado en otras revistas no incluidas en la relación que aquí se emplea. Asumiendo estas eventualidades, la propuesta de análisis puede ayudar a entender, a grandes rasgos, como se han abordado los estudios desde el punto de vista territorial y temático de la Geografía Hispanoamericana en España en los últimos veinticinco años. El universo de análisis se limita a la información disponible a través de bases de datos y revistas indexadas, por lo que no se contempla toda la producción científica existente para el periodo de análisis.

Desde la perspectiva temporal se ha concentrado la atención en el periodo comprendido entre 1990 y 2016. La razón de la fecha inicial se explica por diferentes razones, algunas de carácter estructural y otras vinculadas a lógicas locales, centradas en coyunturas específicamente españolas. De carácter global es el hecho de que desde finales de los años ochenta del siglo XX nuestra sociedad haya entrado en un periodo en el que han cobrado un protagonismo creciente y tremendamente acelerado las nuevas tecnologías de la información y la comunicación (Milton Santos definía este periodo en los años noventa del siglo pasado como científico-técnico-informacional) (Santos, M., 1996). En este escenario de cambio han aparecido nuevos modelos y formatos de difusión de la producción científica, en los que Internet se ha convertido en una herramienta potente y casi instantánea de relación y difusión. Es desde mediados de los años noventa cuando las revistas geográficas españolas comienzan un proceso de ajuste y cambio, al que se vienen incorporando con desigual velocidad cada una de ellas, pasando del formato único de edición impresa (que tiene una tirada reducida de ejemplares y un proceso lento y limitado de distribución, que se centra generalmente en bibliotecas especializadas), a convivir con la aparición de las publicaciones digitales, más económicas y de mucho mayor alcance. Esto explica que desde finales del siglo XX ya existan algunas publicaciones en formato de revista virtual (es el caso de Scripta Nova, que comenzó su andadura digital en 1997), al que han seguido otras muchas desde principios del siglo XXI.

Dentro de las lógicas locales españolas, hay que apuntar que desde comienzo de la década de los años noventa se ha prestado una mayor atención en España al avance científico hispanoamericano en diferentes disciplinas y, entre ellas, en la Geografía. La razón está en los esfuerzos institucionales por conmemorar el Quinto Centenario, con la consecuente atención a este territorio. Esto llevó asociado una mayor disponibilidad de recursos para abordar trabajos de investigación apoyados por algunos programas institucionales del Gobierno, de las comunidades autónomas y de las universidades españolas que, a finales del siglo $\mathrm{XX}$, animaron las investigaciones y estudios geográficos sobre Hispanoamérica (Panadero, 2004: 286). Como resultado de todo ello se ha incrementado el interés de la Geografía por estudiar territorios y temática de muchos ámbitos iberoamericanos, y en este proceso han participado autores de ambos lados del Atlántico (Panadero, 2004: 279).

En este caso, el trabajo de búsqueda y organización temática de las publicaciones se ha realizado en el Centro de Estudios Territoriales Iberoamericano de la Universidad de Castilla-La Mancha (España), que cuenta con un observatorio de la producción de la Geografía española sobre Iberoamérica. Para ello se ha hecho una propuesta metodológica de búsqueda y organización de las referencias bibliográficas por regiones, países, temas y subtemas. El trabajo se viene desarrollando desde el año 2012, y ya ha dejado algunos resultados parciales (Cebrián, 2013). La información se ha generado sobre una estructura en formato de base de datos en la que se han 
introducido las referencias bibliográficas reproducidas en las revistas seleccionadas. Los criterios de clasificación se han estructurado en dos escalas de análisis. El primero responde a un ámbito regional supraestatal, que incluye las categorías que suelen aparecer en catálogos de organismos internacionales (Brasil y Guayana; Caribe; Cono Sur; México y Centroamérica; Países Andinos; a estos cinco se ha agregado una sexta categoría para el conjunto de Hispanoamérica). El segundo se ha efectuado a nivel de país (lo que supone que lo elaborado por debajo del ámbito nacional se adscribe a esta unidad), incluyendo veintiuna categorías, correspondientes a los países de mayor peso territorial y demográfico: Argentina, Bolivia, Brasil, Chile, Colombia, Costa Rica, Cuba, Ecuador, El Salvador, Guatemala, Guayana, Honduras, México, Nicaragua, Panamá, Paraguay, Perú, Puerto Rico -se incluye este estado libre asociado a EEUU porque territorialmente se encuentra dentro del ámbito iberoamericano-, República Dominicana, Uruguay, Venezuela. Se han excluido los estados menores, que no están representados en los estudios realizados o se han analizado integrándolos dentro de sus respectivas áreas geográficas supraestatales.

Desde el punto de vista de las temáticas de los trabajos, se han establecido dos criterios de organización. Por una parte, se han clasificado a partir de las principales ramas de pensamiento y trabajo: Geografía General, Geografía Física, Geografía Humana, Estudios Regionales, Pensamiento Geográfico, Didáctica de la Geografía, Teledetección y SIG. Por otro lado, se ha establecido un segundo nivel de análisis en sub-áreas temáticas, con un mayor nivel de precisión y desagregación: Biogeografía, Climatología, Geografía de la Población, Geografía de las Actividades Económicas, Geografía del Transporte; Geografía del Turismo, Ocio y Recreación; Geografía Industrial; Geografía Política; Geografía Rural; Geografía Urbana; Geomorfología; Medio Ambiente; Paisaje; Riesgos Naturales; a todos ellos se ha añadido otra categoría que recoge aquellos trabajos que nos adscriben explícitamente a ninguna de las categorías anteriores.

\section{La producción científica sobre la Geografía Hispanoamericana en España a partir de las revistas}

El resultado del análisis ha permitido identificar un nutrido conjunto de trabajos reproducidos en las revistas españolas. Han sido un total de 1.661 los encontrados y extraídos, en su mayor parte elaborados por geógrafas y geógrafos españoles. Pero también hay muchos de ellos escritos por autores extranjeros, en su mayor parte de países iberoamericanos.

De la revisión destaca el hecho de que todas las revistas dedican una parte de sus estudios y repertorios de trabajo al ámbito territorial hispanoamericano con numerosos artículos sobre América Latina y el Caribe. Esto pone de manifiesto la decidida voluntad de los consejos de redacción por abrir sus horizontes editoriales al espacio iberoamericano. Pero es también muy ilustrativo el acusado desequilibrio existente en cuanto a número de trabajos en cada una de ellas. Mientras un grupo reducido aparece muy bien posicionado, con una gran cantidad de artículos de investigación, en otros casos son pocos los que se incluyen y en algunas de ellas su presencia es casi testimonial. Las razones de estos desequilibrios son diversas, y no es objeto de este trabajo identificarlas, aunque sí que se pueden aventurar las causas principales. Por un lado, algunas revistas tienen una temática muy especializada (e. g. turismo, cartografía y teledetección, ciudades y procesos urbanos) y dan cabida a trabajos de diferentes territorios, incluidos los de Hispanoamérica (Olcina y Cantos, 2004: 150). En otros casos se debe a que sus estrategias editoriales han facilitado el 
proceso de internacionalización de los artículos publicados, aprovechando las oportunidades que ofrecen las nuevas tecnologías de la información. No hay que olvidar la posición que ocupan en los rankings de publicaciones periódicas y el efecto llamada que tiene este hecho sobre autores de procedencia diversa.

\section{La distribución de los trabajos por revistas}

Como se apuntaba con anterioridad, un hecho que llama poderosamente la atención es el desigual reparto que se produce entre las revistas consultadas. Se pueden identificar dos grupos a partir de presencia de trabajos sobre el ámbito hispanoamericano, que aglutinan la mayor parte de la producción. El primero está formado por las que cuentan con una muy elevada presencia (más de cien trabajos en su repertorio editorial). Dentro de esta primera categoría están: Scripta Nova (195 artículos), Mapping (190 trabajos), Biblio 3W (188), Ciudad y Territorio-Estudios Territoriales (134) y Estudios Geográficos (111). Entre las cinco suman 818 artículos, lo que supone casi la mitad de lo producido $(49,2 \%)$. Se trata de revistas de procedencia diversa, ya que algunas dependen de organismos públicos de ámbito estatal, o forman parte de Departamentos universitarios e institutos de investigación. Todas están bien posicionadas en los rankings internacionales, cuentan con una larga tradición, tienen una elevada especialización temática o han apostado como línea estratégica por la difusión a través de las nuevas tecnologías de la información y la comunicación.

En una segunda categoría se han recogido aquellas que se encuentran entre los 30 y los 100 trabajos publicados (se ha empleado el umbral 30 porque se corresponde con la media entre el total de artículos recogidos -1.661- y las revistas incluidas en la serie -56-). En esta segunda categoría aparecen diez revistas: Anales de Geografía de la Universidad Complutense (87), Cuadernos Geográficos de la Universidad de Granada (79), Geofocus (63), Cuadernos de Turismo (56), Boletín de la Asociación de Geógrafos Españoles (44), Ería (40), Investigaciones Geográficas (38), Papeles de Geografía (38), Geographicalia (36) y Documents d'Analisi Geográfica (33). En total son 514 los artículos recogidos en esta segunda categoría, lo que supone el 30,9\% del total.

Estos dos grupos concentran la mayor parte de la producción científica sobre lberoamérica. Las quince revistas acaparan el $80 \%$ del total publicado (1.332 trabajos). En su mayor parte están vinculadas a las universidades mayores y más consolidadas, y a Departamentos de Geografía con una larga tradición. También aparecen algunas pertenecientes a asociaciones de carácter eminentemente disciplinar en Geografía. Son las mejor posicionadas en los rankings nacionales e internacionales de acuerdo a los parámetros estandarizados de calidad. El resto cuentan con una presencia menor, que en algunos casos apenas es testimonial.

\section{La evolución temporal de los trabajos}

Ha sido sobre todo desde la década de los años noventa cuando se ha producido un incremento notable en el número de artículos, aunque desde enfoques temáticos, disciplinares y territoriales variados. La evolución temporal permite establecer varios periodos, algunos de mayor progresión y otros marcados por el descenso. Esta evolución ha estado muy influenciada por determinados contextos de la sociedad y la economía española de las últimas décadas, que han tenido repercusiones claras en la actividad investigadora (Figura $N^{\circ} 1$ ). 
Figura $\mathrm{N}^{\circ} 1$

Evolución temporal de los trabajos publicados en artículos de revistas

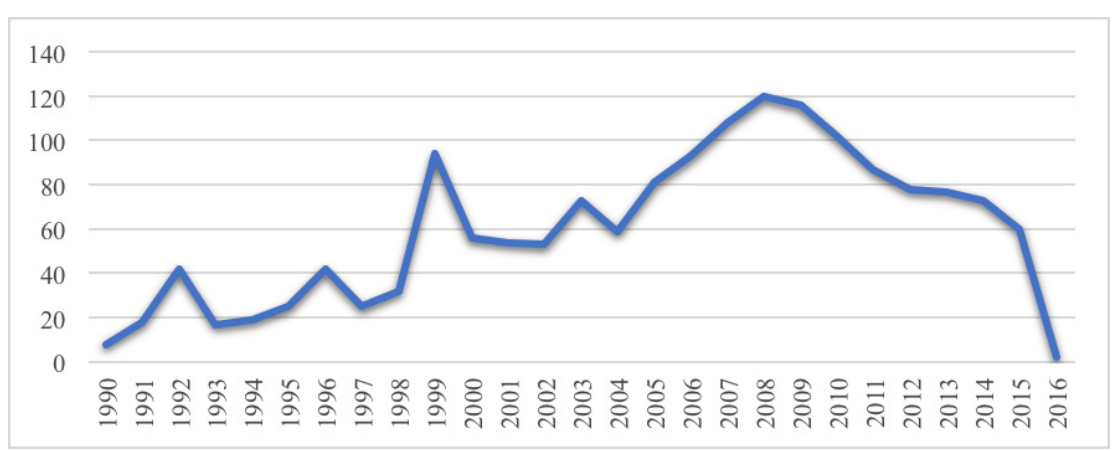

Fuente: Elaboración propia.

\section{La distribución territorial de los artículos: las grandes unidades regionales de atención temática}

Por grandes ámbitos regionales existe una cierta homogeneidad en cuanto a la distribución temática de los artículos. Por una parte, hay muchos trabajos de ámbito continental, lo que pone de manifiesto el interés por la visión de síntesis para el conjunto de Iberoamérica (suponen el $12,3 \%$ del total) (Cuadro $N^{\circ} 2$ ).

Cuadro $\mathrm{N}^{\circ} 2$

Distribución de los artículos por grandes unidades regionales

\begin{tabular}{|l|r|r|}
\hline Región & Frecuencia & $\%$ \\
\hline América Latina & 205 & 12,3 \\
\hline Brasil y Guayana & 258 & 15,5 \\
\hline Caribe & 256 & 15,4 \\
\hline Cono Sur & 467 & 28,1 \\
\hline México y Centroamérica & 328 & 19,7 \\
\hline Países andinos & 144 & 8,7 \\
\hline Sin definir & 3 & \\
\hline Total general & 1.661 & \\
\hline
\end{tabular}

Fuente. Elaboración propia, diciembre 2016

Es significativo el hecho de que, a partir de las agrupaciones regionales definidas, el Cono Sur (Argentina, Uruguay, Paraguay y Chile) aparece como el principal espacio de interés, y es ahí donde se concentra más de la cuarta parte de los artículos publicados (28,1\%), seguido por México y Centro América (19,7\%). Estos dos ámbitos antes mencionados recogen casi la mitad de los trabajos producidos. En tercera y cuarta posición quedan, en una situación de cierto equilibrio entre ellos, Brasil y Caribe (15,5\% y 15,4\% respectivamente). Finalmente aparece la región andina (Colombia, Venezuela, Ecuador, Perú y Bolivia) como espacio de menor atención. 


\section{La distribución por países}

Si a escala de región se aprecia un cierto equilibrio, destaca la elevada polarización a nivel de países. Hay que tener presente que no todos los trabajos tienen un solo Estado como objeto de atención, lo que de alguna manera obliga a considerar los resultados con una cierta cautela. Pese a ello aparecen algunos a los que se ha dirigido un mayor interés. Entre los territorios que cuentan con un protagonismo elevado, con más de 75 trabajos publicados (se ha utilizado la media -valor 75-, sobre el total de los veintiún países considerados y los 1.661 registros identificados), quedan seis categorías de referencia: México (261), Argentina (321), Chile (122), Cuba (224), Brasil (256), a lo que se suma otra que incluye el conjunto de Hispanoamérica (169).

En un segundo grupo, entendido como de interés alto, aunque por debajo de la media (en esta categoría se incluyen los comprendidos entre 30 y 75 casos), aparecen Perú (41), Venezuela (38) y Colombia (32). Existen menos trabajos por razones diversas, entre las que destacan la menor presencia de estudios de geografía, lo que limita la producción y las relaciones. No obstante, hay que apuntar que la importancia territorial, demográfica y económica de los países más representados actúan como principal factor explicativo. Pero también se puede entender por la mayor tradición de la disciplina geográfica en estos territorios. Se suman a ello las relaciones crecientes entre universidades españolas e iberoamericanas que ayudan a entender esta situación.

\section{Los grandes ámbitos temáticos de interés}

Se han agrupado los trabajos por grandes ámbitos y sub-ámbitos temáticos. A nivel de ámbito, la atención se ha repartido con una cierta homogeneidad entre las diferentes categorías establecidas, aunque con matices y mayor atención hacia algunas de ellas. La Geografía Humana ha suscitado un interés muy superior al resto (supone más del $40 \%$ del total). En el otro extremo aparecen dos categorías con presencia sustancialmente menor: se trata de la Didáctica de la Geografía, en la que apenas se han publicado 41 trabajos (menos del 3\%) y Pensamiento Geográfico, con 100 trabajos (6\%). En los otros tres grupos, en posiciones intermedias, aparecen valores relativamente uniformes, que oscilan entre $173(10,4 \%)$ en el caso de los estudios de carácter regional, 226 en estudios de carácter general, y 234 para los de Geografía Física (Figura № 2).

Figura $\mathrm{N}^{\circ} 2$

Distribución de los artículos por grandes ámbitos temáticos de interés

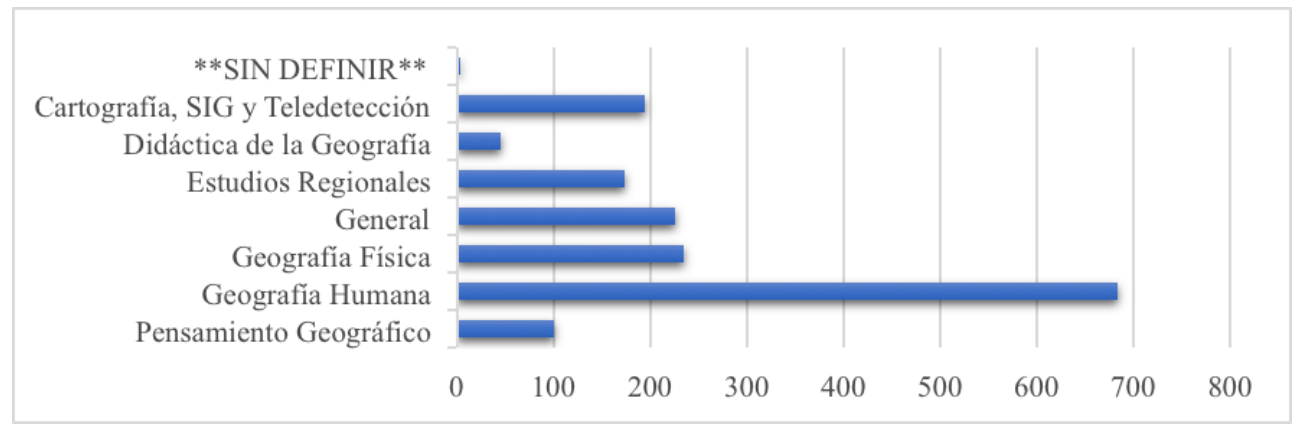

Fuente: Elaboración propia. 
Se han analizado también los trabajos desde sub-ámbitos temáticos. Hay en este criterio un número mayor de categorías (14 en total). Es interesante observar como aparecen discrepancias sustanciales entre ellas, con algunas muy representadas, frente a otras en las que la atención ha sido menor, y en ocasiones apenas testimonial. La mayor incidencia, con diferencia, se ha concentrado en algunas de las disciplinas de la Geografía Humana, y en especial la Urbana.

Figura $\mathrm{N}^{\circ} 3$

Distribución de los artículos por grandes sub-ámbitos temáticos de interés

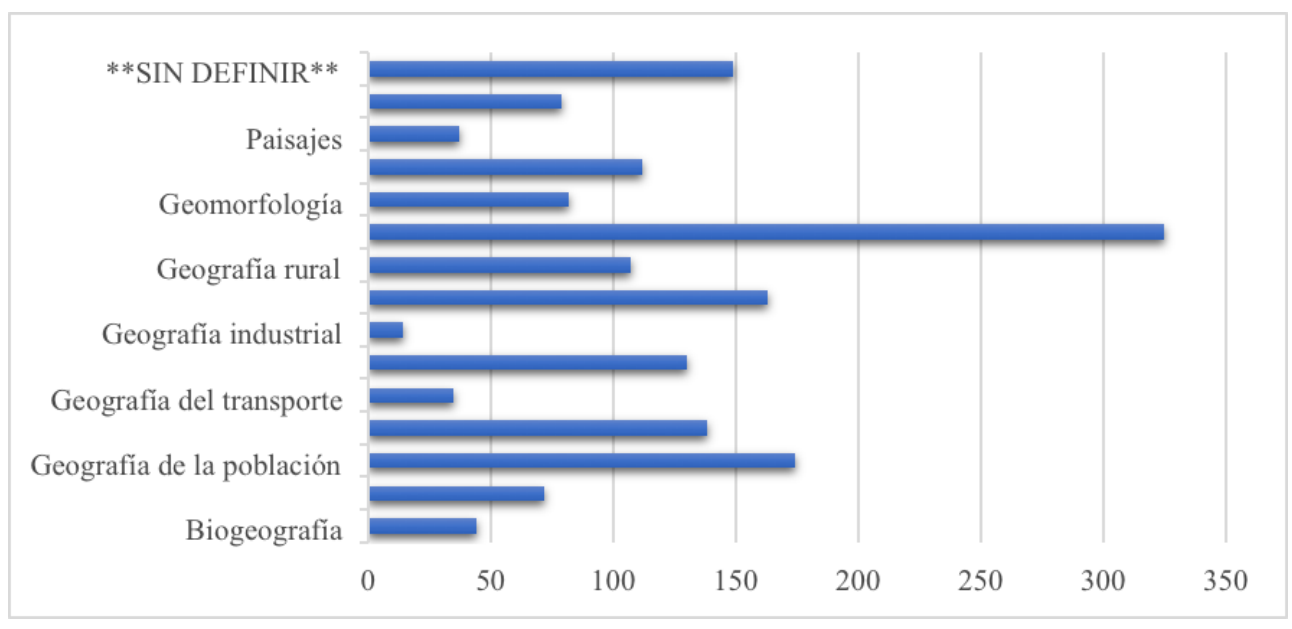

Fuente: Elaboración propia.

Le sigue en importancia la de la Población, la Política, la de las Actividades Económicas y la del Turismo y el Ocio, que por su importancia se ha considerado como un campo aparte. También los estudios relacionados con el Medio Ambiente y la Geografía Rural (en estas siete categorías, que son las que cuentan con más de cien trabajos, se concentran en $70 \%$ de la producción). El resto están menos presentes, con la singularidad de que la Geografía industrial (14), o los estudios de paisaje (37) apenas cuentan con un reducido número de autores y trabajos interesados en estas temáticas (Figura $\mathrm{N}^{\circ} 3$ ).

\section{La producción científica sobre la Geografía Iberoamericana en España a partir de las tesis doctorales}

El segundo criterio de análisis empleado es la producción de tesis doctorales. En este caso las características de los trabajos son bien diferentes a los artículos de investigación. Se trata de estudios de mayor profundidad y que requieren periodos de tiempo más dilatados en su elaboración. Por otro lado, están asociadas a programas de doctorado, lo que supone requerimientos académicos específicos, y, sobre todo, una vinculación del doctorando con una universidad de referencia. Hacen falta también directores que reúnan los requisitos que establece la legislación, y sobre todo temáticas que tengan afinidad entre las líneas de investigación de directores y doc- 
torandos. En este escenario es el que hay que contextualizar la defensa de tesis doctorales en España sobre América Latina. Para analizarlas se ha realizado un seguimiento dentro de la base de datos Teseo (https://www.educacion.gob.es/Teseo), identificando aquellas cuya temática está específicamente dedicada al territorio latinoamericano. Se han localizado 176 en el periodo comprendido entre 1990 y 2016.

\section{La distribución de las tesis doctorales por universidades}

Una primera circunstancia a tener en consideración es la distribución por universidades. Llaman la atención varios hechos. Por una parte, el que la mayor parte de las universidades españolas hayan tenido presencia en este proceso. Han sido treinta y una las que han participado formando doctores. El otro es la elevada polarización en algunas universidades. Destaca el papel desarrollado por la Universidad de Barcelona, que ha concentrado la práctica totalidad de las tesis defendidas en Cataluña: de 35 tesis doctorales (19,8\% del total), 28 se han hecho en esta Universidad. Es la que ha liderado el proceso y la que ha tenido una mayor presencia a escala nacional. En el caso de la Comunidad de Madrid ha existido un mayor equilibrio y reparto, ya que hay tres universidades que actúan como principales referentes: la Complutense de Madrid, con 19 tesis doctorales (10,8\%); la de Alcalá de Henares, con 17 (9,7\%); y la Autónoma de Madrid con 11 (6,2\%). En el caso de las universidades andaluzas ha sido la de Sevilla la que tenido un protagonismo indiscutido (19 tesis defendidas - 10,8\%-). Por debajo de estas, y en posiciones más alejadas, pero con un cierto equilibrio entre ellas, aparecen varias: en el caso de la Universidad de León han sido 7 (4\%), y con 4 (2,3\%) tesis aparecen Cantabria, Islas Baleares, Las Palmas de Gran Canaria, Oviedo, Santiago de Compostela o Castilla-La Mancha.

\section{Evolución de las tesis doctorales defendidas en España}

Es interesante analizar la evolución temporal de los trabajos. Como consideración general se puede apuntar un cierto paralelismo con lo que ha sucedido en el caso de los artículos publicados en revistas de investigación. Se pueden identificar dos momentos bien diferenciados. El primero se corresponde con la década de los años noventa, en la que la dinámica ha venido marcada por la estabilidad y una escasa presencia de trabajos de investigación de esta naturaleza, donde los pocos aparecidos tenían un carácter casi pionero. Lo habitual ha sido que cada año se defendieran uno o dos trabajos (Figura $\mathrm{N}^{\circ} 4$ ).

A partir del año 2000 se aprecia un cambio significativo. Las razones posiblemente son diferentes. Ninguna de ellos puede entenderse a título individual y seguramente se puede explicar por la conjunción de todas ellos. Por un lado, está la aparición de nuevos Grados, en sustitución de las antiguas licenciaturas, con nuevos programas de doctorado vinculados a las estructuras académicas (Real Decreto 56/2005, de 21 de enero, por el que se regulan los estudios universitarios oficiales de Posgrado; Real Decreto 99/2011, de 28 de enero, por el que se regulan las enseñanzas oficiales de doctorado). Por otra parte, están las relaciones institucionales entre universidades, gestadas en buena medida a lo largo de la década de los años noventa, con la apertura de líneas de investigación con la Geografía Iberoamericana como objeto de atención. Finalmente está el hecho de que en estos años ha habido una mayor disponibilidad de recursos para la movilidad del alumnado y el profesorado, en un contexto de crecimiento económico, que ha favorecido este proceso. Aparece en este segundo periodo un aumento generalizado, con algunos años en los que se produce una cantidad sorprendentemente elevada de trabajos: 15 en 2005; 19 en 2013 y 
22 en 2016. Posiblemente estos picos estén vinculados a coyunturas específicas, como cambios en las normativas o en los programas de doctorado. Sea cual fuere el motivo, lo evidente es que desde el año 2000 se ha incrementado la producción científica.

Figura $\mathrm{N}^{\circ} 4$

Evolución del número de tesis doctorales defendidas en España

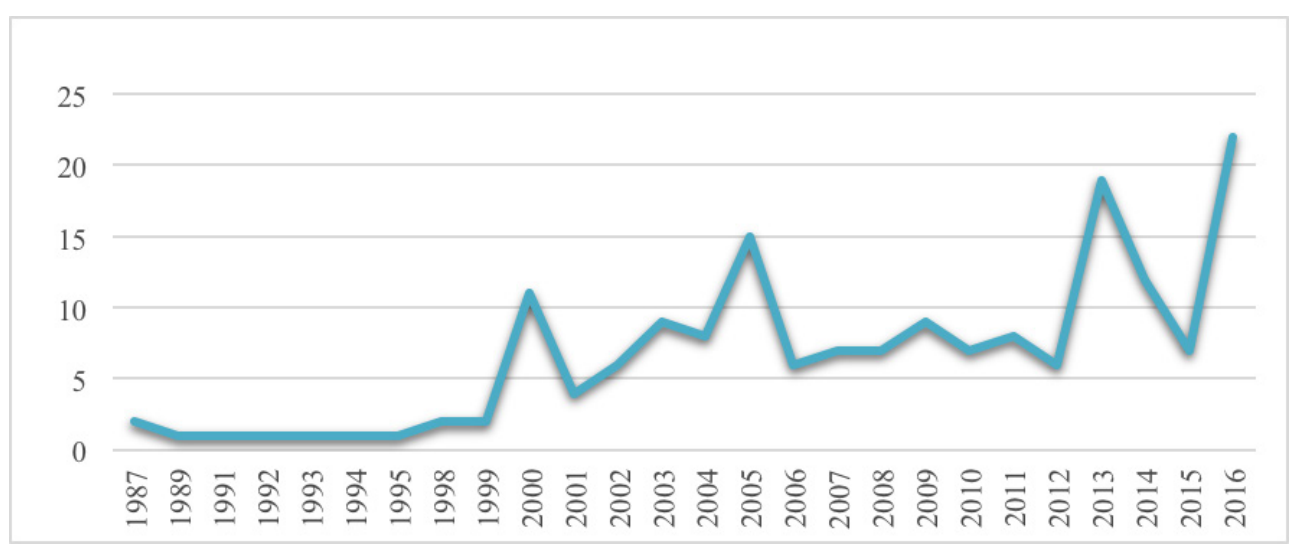

Fuente. Elaboración propia a partir de Teseo.

\section{La distribución territorial de las temáticas de las tesis doctorales: las grandes unidades regionales de atención}

A escala de grandes ámbitos territoriales se observan desequilibrios importantes, con tres áreas de atención prioritaria. El primero de ellos se centra en los países andinos, que aglutinan la cuarta parte de las tesis doctorales elaboradas. En segunda posición se encuentra Brasil, seguido de Centroamérica y México. En cuarta posición está el conjunto de los países del Cono Sur (Cuadro $\left.N^{\circ} 3\right)$.

\section{Cuadro $\mathrm{N}^{\circ} 3$}

Distribución de las Tesis Doctorales por grandes unidades regionales

\begin{tabular}{|l|l|l|}
\hline Región & Frecuencia & $\%$ \\
\hline América Latina & 5 & 2,86 \\
\hline Brasil y Guayana & 43 & 24,57 \\
\hline Caribe & 4 & 2,28 \\
\hline Cono Sur & 30 & 17,14 \\
\hline México y Centroamérica & 14 & 8 \\
\hline México y Centroamérica & 35 & 20 \\
\hline Países andinos & 44 & 25,14 \\
\hline Total & 175 & 100 \\
\hline
\end{tabular}

Fuente: Elaboración propia a partir de Teseo. 


\section{La distribución por países}

Por países aparecen también algunos aspectos relevantes. Por un lado, la desigual presencia de cada uno de ellos. Por otro, la fuerte concentración en un grupo reducido.

Figura $\mathrm{N}^{\circ} 5$

Distribución de las tesis doctorales por países

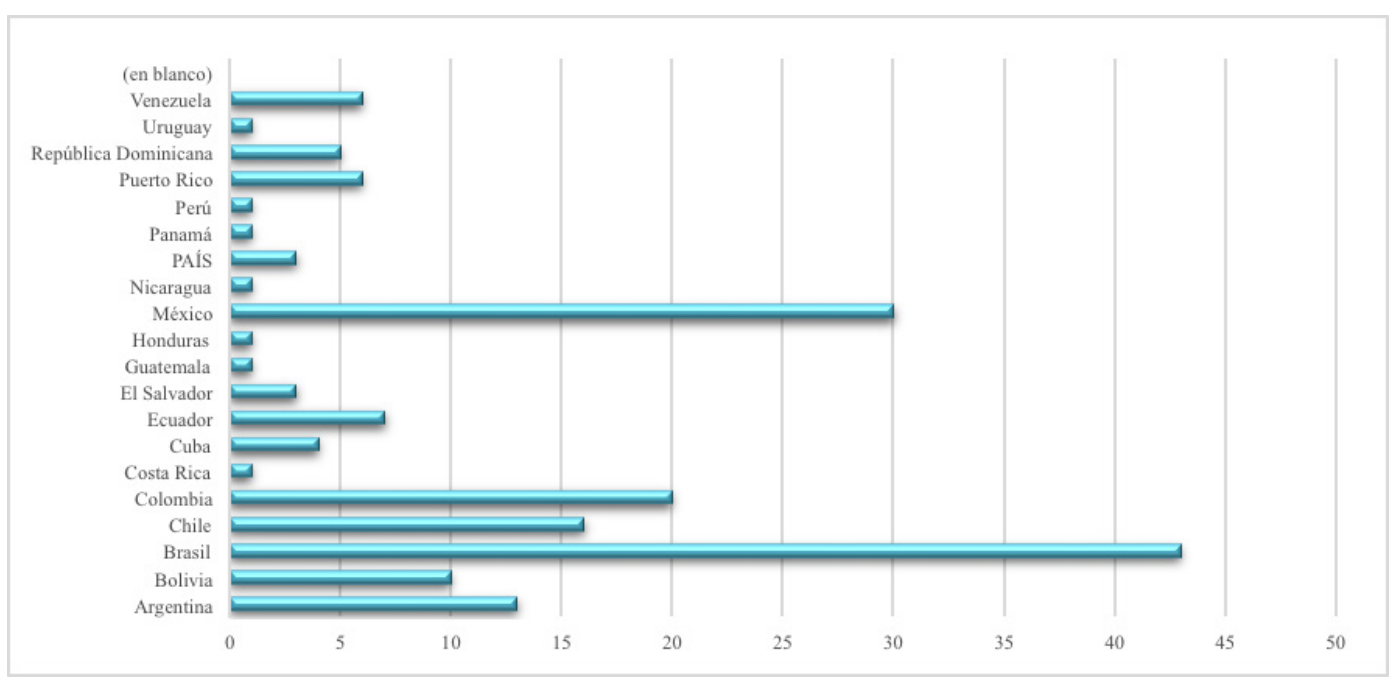

Fuente. Elaboración propia a partir de Teseo.

Llama la atención la presencia especialmente significativa de las tesis doctorales realizadas sobre Brasil. Prácticamente el $25 \%$ de los trabajos se han dedicado a analizar diferentes aspectos sobre este país. Le sigue en importancia México, con casi un $17 \%$ de las tesis doctorales defendidas. Por detrás se encuentran Colombia (11,5\%), Chile (9,2\%), Argentina (7,5\%) y Bolivia (5,7\%). En el resto la presencia es más reducida (Figura $\mathrm{N}^{\circ} 5$ ).

\section{Los grandes ámbitos temáticos de interés}

Por grandes ámbitos temáticos, existe un desequilibrio manifiesto, ya que la mayor parte de las tesis doctorales se adscriben al ámbito de la Geografía Humana, donde se concentra el 48\%. Le sigue en importancia los estudios regionales (20\% de las tesis) y la Geografía Física (17,7\%). Una más reducida trascendencia tiene el conjunto de trabajos de Cartografía, SIG y Teledetección $(7,4 \%)$ (Cuadro $\left.N^{\circ} 4\right)$. 
Cuadro $\mathrm{N}^{\circ} 4$

Distribución de las Tesis Doctorales por grandes ámbitos temáticos

\begin{tabular}{|l|l|l|}
\hline Ámbitos & Frecuencia & $\%$ \\
\hline Cartografía, SIG y Teledetección & 13 & 7,4 \\
\hline Didáctica de la Geografía & 8 & 4,6 \\
\hline Estudios Regionales & 35 & 20 \\
\hline Geografía Física & 31 & 17,7 \\
\hline Geografía Humana & 84 & 48 \\
\hline Pensamiento Geográfico & 4 & 2,3 \\
\hline Total general & 175 & \\
\hline
\end{tabular}

Fuente: Elaboración propia a partir de Teseo.

Al analizar los sub-ámbitos temáticos se advierten también desequilibrios notorios, ya que una parte considerable están dedicado a la Geografía Urbana, que es, sin lugar a dudas, el principal objeto de atención e investigación. Destacan también los trabajos dedicados al estudio de la población y a la Geografía Rural. Por detrás queda la Geografía del Turismo y la Económica, las dirigidas a analizar procesos ambientales o la Biogeografía (Figura $\mathrm{N}^{\circ} 6$ ).

Figura $\mathrm{N}^{\circ} 6$

Distribución de las tesis doctorales por sub-ámbitos temáticos

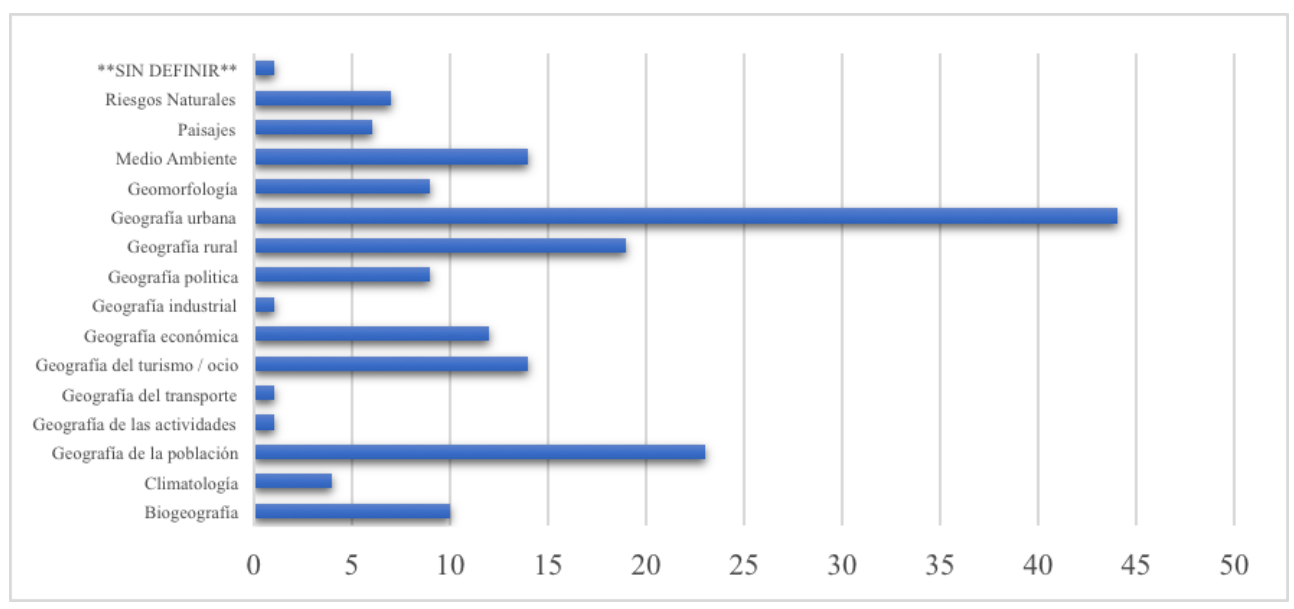

Fuente: Elaboración propia a partir de Teseo.

\section{Conclusiones}

A partir de la década de los noventa del siglo XX se han producido cambios de gran calado que han impulsado los estudios de Geografía en España sobre Iberoamérica. Fundamental ha sido el desarrollo de las TICs, con Internet y nuevos formatos de revistas. También ha aumentado 
la movilidad de la población en un contexto de relaciones crecientes y de economía expansiva, lo que ha favorecido el interés por territorios de origen y destino de relaciones personales y económicas. Por otro lado, ha habido una apuesta decidida a nivel institucional por favorecer este tipo de relaciones académicas y de conocimiento, y por impulsar la producción científica sobre Iberoamérica, en la que han participado la administración nacional, las autonómicas y las propias universidades. Estas razones han sido bien recibidas en la comunidad académica, y se han abierto numerosas vías de relación desde ambos lados del Atlántico. Como resultado han aparecido alianzas estratégicas, proyectos conjuntos, programas de formación integrados... En definitiva, se han multiplicado las conexiones y han aumentado los resultados, que se han producido bajo formatos diferentes, pero muchos de ellos editados como artículos de revistas y como tesis doctorales.

Desde el punto de vista de la Geografía española, se trata posiblemente de uno de los mayores cambios acontecidos en los ámbitos territoriales de atención y estudio, ya que hasta la década de los noventa los trabajos elaborados desde esta disciplina sobre Iberoamericana apenas tenían un interés marginal. Es a partir de esos años cuando se produce un despertar y un crecimiento importante, que se ha acentuado con el cambio de milenio y se ha mantenido hasta 2008, en un contexto económico e institucional favorable, y que ha dejado como resultado numerosos artículos en revistas y la defensa de un nutrido grupo de tesis doctorales. A partir de ese momento se ha roto la tendencia, con un descenso preocupante, ya que en el año 2015 se registraba una situación similar a la de 1998.

El proceso ha sido, además de progresivo, selectivo tanto por las universidades en las que se ha incrementado la atención como por en los territorios objeto de análisis. Algunas universidades han actuado como catalizadoras del proceso. Han sido las más consolidadas y han actuado acompañadas de asociaciones con una dilatada tradición en los estudios de Geografía: la de Barcelona y las madrileñas, junto con la AGE (Asociación de Geógrafos Españoles) han liderado un proceso al que se han ido incorporando otras universidades más jóvenes o menos dimensionadas. También han aparecido desequilibrios en la orientación de los trabajos a escala de regiones, países y temáticas. Las desigualdades hay que asociarlas posiblemente a la diferente implantación de la disciplina en enfoques y producción científica. Los países del Cono Sur, Brasil y México han tenido un protagonismo indiscutido, junto con Cuba. Son los territorios más poblados, pero también los que han dejado una disciplina geográfica más proactiva. Los trabajos han sido especialmente numerosos dentro del ámbito de la Geografía Humana y, dentro de ésta, en la Urbana, que ha polarizado buena parte de la atención, aunque también es cierto que han estado presentes prácticamente todas las corrientes disciplinares, pero con desigual protagonismo. En el caso de las tesis doctorales ha existido un cierto paralelismo, ya que han ido creciendo en número, especialmente con el cambio de milenio, y se ha producido con una diferenciación territorial mayor, aunque con una cierta similitud en las orientaciones temáticas y disciplinares, sin que se haya roto la tendencia.

Por otro lado, se ha realizado una apuesta decidida por parte de muchas revistas españolas por modernizar sus formatos y, sobre todo, por incrementar su internacionalización. La mayor parte de ellas (sobre todo las más implantadas y mejor posicionadas) han realizado esfuerzos notables por incorporar trabajos de países diferentes, pero con especial atención hacia lberoamérica, y temáticas distintas. 
A la vista de lo recogido en estas páginas, parece evidente que los esfuerzos académicos (e.g. administraciones, universidades, departamentos de Geografía) han producido frutos muy positivos. Estamos siendo actores de un creciente proceso de integración de la disciplina, que ha abierto canales de relación a través de proyectos formativos o investigadores, cuyos resultados aparecen reproducidos como artículos y tesis doctorales. Pero es necesario reflexionar sobre los efectos de la falta de acompañamiento institucional en periodos de crisis o de recesión. También es necesario reflexionar sobre los efectos que tienen los soportes utilizados en el proceso de creación y difusión del conocimiento en el ámbito de la Geografía.

Queda por tanto mucho camino por recorrer y, sobre todo, se hace necesario mantener vivos los lazos establecidos en el último cuarto de siglo, especialmente porque el proceso es bidireccional ya que existen revistas de Geografía en Chile, Argentina, Brasil o México (entre otras) que acogen trabajos producidos sobre la Geografía en España. Crecen los puentes y se incrementan las relaciones.

\section{Referencias bibliográficas}

CABERO, V. Iberoamérica, en el horizonte investigador y en el quehacer geográfico español. Boletín de la Asociación de Geógrafos Españoles, 1993, № 15-16, p. 3-12.

CAPEL, H. La Geografía Española tras la Guerra Civil. Revista Geocrítica. Cuadernos Críticos de Geografía Humana. Barcelona: Universidad de Barcelona, 1976.

CARPIO, J. Informe sobre la investigación en el Grupo de Trabajo de Geografía de América Latina en la Geografía Española. En: LASANTA, T. y MARTÍN VIDE, J. (coordinadoras). La investigación geográfica en España (1990-2012). Jaca: AGE, Instituto Pirenaico de Ecología (CSIC), 2013, p. 401-412.

CEBRIÁN, F. Las aportaciones de las revistas españolas a la Geografía Iberoamericana en el siglo XXI. Boletín de la Asociación de Geógrafos Españoles (BAGE), 2013, Nº63, p. 519-533.

ESPEJO, C. América Central y el Caribe en las revistas y congresos de Geografía españoles. Revista Geográfica de América Central, 1997, №34.

GARCÍA, J.C.; MICHELINI, J.J. y MINGUEZ, C. La presencia de la Geografía iberoamericana en las revistas científicas españolas. Biblio 3W. Revista Bibliográfica de Geografía y Ciencias Sociales. Universidad de Barcelona, 2009. Disponible en Internet:

http://www.ub.edu/geocrit/b3w-845.htm

GÓMEZ, J. La evolución de la investigación de la Geografía española durante los últimos decenios. En: LASANTA, T. y MARTíN VIDE, J. (coordinadoras). La investigación geográfica en España (19902012). Jaca: AGE, Instituto Pirenaico de Ecología (CSIC), 2013, p. 21-42.

GURRIA, J.L.; NIETO, A. y CÁRDENAS, G. La producción científica de la Geografía española en Iberoamérica. En: GÓMEZ MENDOZA, J. La evolución de la investigación geográfica española durante los últimos decenios. Madrid: AGE, 2013, p. 189-204. 
GUTIERREZ, J. Las revistas internacionales de Geografía: internacionalización e impacto. Boletín de la Asociación de Geógrafos Españoles, 1999, № 27, p. 117-134.

MENDOZA, H. Las oportunidades y desafíos del siglo XXI para la geografía latinoamericana. Biblio 3W. Revista Bibliográfica de Geografía y Ciencias Sociales, 2001, Nㅜ285, Universidad de Barcelona.

OLCINA, J. y ROMÁN, A. Las revistas españolas de Geografía. Cambios y adaptación a los criterios editoriales de calidad. En: La geografía española ante los retos de la sociedad actual. Aportación española al XXX Congreso de la UGI. Madrid: Glasgow Editorial Mayoral, AGE y RSG., 2004, p. 145179

PANADERO, M. La internacionalización de la Geografía española. Los intercambios con la Geografía y los geógrafos de América Latina. En: Comité Español de la UCI: La Geografía española ante los retos de la sociedad actual. Aportación española al XXX Congreso de la Unión Geográfica Internacional. Madrid: Glasgow Editorial Mayoral, AGE y RSG., 2004, p. 279-298 y p. 669-678.

SANTOS, M. De la totalidad al lugar. Barcelona: Oikos-Tau, 1996.

VALENZUELA, M. La diversidad en la bibliográfica geográfica sobre España, una aproximación al periodo 1990-2000. En: Vivir la diversidad en España. Aportación española al XXIX Congreso de la UGI. Madrid: Asociación de Geógrafos Españoles, Real Sociedad Geográfica y Caja Duero, 2000, p. 357-372.

VALENZUELA, M. Las universidades españolas, de la Dictadura al Estado autonómico. Difusión espacial y regeneración urbana. En: VALENZUELA, M. (coordinación). El impacto del modelo autonómico en las ciudades españolas. Una aproximación interdisciplinar. Madrid: Servicio de Publicaciones de la Universidad Autónoma de Madrid, 2013. 
